\title{
MULTIWAVELENGTH STUDY OF THE WO STAR SAND 2
}

\author{
L. NORCI ${ }^{1}$, V.F. POLCARO ${ }^{2}$, C. ROSSI ${ }^{3}$ and R. VIOTTI ${ }^{2}$ \\ ${ }^{1}$ Max-Planck-Institut für Extraterrestrische Physik, Postfach 1603, D-8574O Garching, \\ B.R. Deutschland \\ ${ }^{2}$ Istituto di Astrofisica Spaziale, via Enrico Fermi 21, I-00044 Frascati, Italy \\ ${ }^{3}$ Istituto Astronomico, Universitá di Roma La Sapienza, I-00161 Roma ,Italy
}

\begin{abstract}
We present the results of a multifrequency observation of the WO star Sand 2 in the LMC. Two ROSAT PSPC observations, with exposure times of $8953 \mathrm{~s}$ and $4540 \mathrm{~s}$, are used to investigate possible $\mathrm{X}$-ray emission from Sand 2. The optical spectrum observed with the ESO $2.2 \mathrm{~m}$ telescope close to the time of the ROSAT observations, shows strong emission lines of $\mathrm{HeII}, \mathrm{CIV}, \mathrm{OV}$ and $\mathrm{OVI}$. All lines have a velocity broadening of $4200 \pm 100 \mathrm{~km} \mathrm{~s}^{-1}$. The UV spectrum from the IUE archive shows a CIV resonance doublet at $155 \mathrm{~nm}$ with a P-Cygni absorption at $\sim-5000 \mathrm{~km} \mathrm{~s}^{-1}$, with wings extending to about $-8000 \mathrm{~km} \mathrm{~s}^{-1}$. A combination of two different $\mathrm{BB}$ temperatures $(80000 \mathrm{~K}$ and $25000 \mathrm{~K})$ are required to fit the UV-optical continuum, dereddened by $E_{B-V}=0.25$.
\end{abstract}

Key words: stars: Wolf-Rayet - WO - LMC - individual: Sand 2

Sand 2 (Br 93, Sk-68 145, FD 73) belongs to a small subgroup (about four objects) of the Wolf-Rayet stars, the WO stars, characterized by the presence of extremely strong $O$ VI 381.1-383.4 nm emission and of a very high velocity wind. Although the WR stars are known to be soft X-ray emitters from Einstein Observatory observations (see Pollock 1987) none of the four WO stars is so far known to be a pronounced X-ray source.

Sand 2 lies in the northern part of the LMC 30 Dor nebula, to which it is probably physically associated (Lortet \& Testor 1991). It is embedded in a bright $\mathrm{H}$ II region, with a very complex [O III] structure (Pakull 1991), and a possible far-IR excess (Lozinskaya 1991). The low resolution spectrum of the star has been published by Torres \& Massey (1988).

The optical spectrum of Sand 2 shows strong emission lines of He II, $\mathrm{C} I \mathrm{~V}$, and $\mathrm{Ov}$, with a velocity broadening of $4200 \pm 100 \mathrm{~km} \mathrm{~s}^{-1}$. The very high ionization $O$ VIII $606.4-6.8 \mathrm{~nm}$ doublet is present in narrow emission (FWHM of about $220 \mathrm{~km} \mathrm{~s}^{-1}$ ), much weaker than that in the galactic WO star Sand 4 (Polcaro et al. 1992).

The ultraviolet spectrum is characterized by many emission lines very prominent over the continuum. The strongest is the $\mathrm{C}$ IV doublet at 155 $\mathrm{nm}$ which also presents a P-Cygni absorption violet shifted by about $4800 \mathrm{~km} \mathrm{~s}^{-1}$. The UV-optical continuum energy distribution, corrected for $E_{B-V}=0.25$, is fitted with a combination of two $80000 \mathrm{~K}$ and $25000 \mathrm{~K}$ black-body spectra.

In the 1.0-2.4 keV energy range a complex of two weak sources are present near the position of Sand 2 in both the ROSAT PSPC observations. No 
source is visible in the intermediate $(0.4-1.0 \mathrm{keV})$ and low $(0.1-0.4 \mathrm{keV})$ energy bands. We have determined the position of the two sources by fitting the source photon distribution with a Gaussian profile, after correcting the two images for the boresight effect by means of field sources of known position. We have superposed the two contour maps by using as a reference point the bright source CAL 71 . Sand 2 clearly lies at an intermediate position between the two X-ray sources. Considering the present uncertainty in the position of the two sources, because of the source weakness and our poor knowledge of the instrument response at so large off-axis angles, it is difficult at the moment to establish whether Sand 2 is the optical counterpart of one of the two sources. It should be noted that the SIMBAD map shows no other stars within a radius of $2^{\prime}$ centered on the sources. But we cannot rule out the possibility of other, much fainter optical counterparts.

The background subtracted count-rate for the weakest of the two X-ray sources is $(2.9 \pm 0.6) \times 10^{-3} \mathrm{~s}^{-1}$. At the distance of LMC this corresponds to a luminosity in the $0.1-2.4 \mathrm{keV}$ energy range of $9.5 \times 10^{33} \mathrm{erg} \mathrm{s}^{-1}$, assuming a spectrum with $\log N_{H}=20$ and $\mathrm{kT}=1 \mathrm{keV}$ (see Pollock 1987). The strongest source is about a factor 2 more luminous.

Higher positioning accuracy (PSPC pointed on the source, HRI) and a better detailed study of the field in the visual is required to ascertain whether Sand 2 can be associated with one of the two detected soft X-ray sources.

\section{References}

Dopita, M. A, Lozinskaya, T., Mc Gregor, P.J., Rawling, S.J. 1990, ApJ 351, 563

Lortet, M.-C., Testor, G. 1991, A\&A Suppl. 89, 185

Lozinskaya, T. 1991, in: K.A. van der Hucht \& B. Hidayat B. (eds.), Wolf-Rayet Stars and Interrelations with Other Massive Stars in Galaxies, Proc. IAU Symp. No. 143 (Dordrecht: Kluwer), p. 371

Pakull, M.W. 1991, in: K.A. van der Hucht \& B. Hidayat B. (eds.), Wolf-Rayet Stars and Interrelations with Other Massive Stars in Galaxies, Proc. IAU Symp. No. 143 (Dordrecht: Kluwer), p. 391

Polcaro, V.F., Viotti, R., Rossi, C., Norci, L. 1992, $A \mathscr{G} A$ 265, 563

Pollock, A.M.T. 1987, $A \& A$ 171, 135

Schmutz W. 1991, in: K.A. van der Hucht \& B. Hidayat B. (eds.), Wolf-Rayet Stars and Interrelations with Other Massive Stars in Galaxies, Proc. IAU Symp. No. 143 (Dordrecht: Kluwer), p. 39

Torres, A.V., Massey, P. 1988, ApJ Suppl. 65, 459 